\title{
Confined Magneto-Optical Waves in Graphene
}

\author{
Aires Ferreira, ${ }^{1,2}$ N. M. R. Peres, ${ }^{1,2}$ and A. H. Castro Neto ${ }^{1}$ \\ ${ }^{1}$ Graphene Research Centre and Department of Physics, \\ National University of Singapore, 2 Science Drive 3, Singapore 117542 \\ ${ }^{2}$ Department of Physics and Center of Physics, University of Minho, P-4710-057, Braga, Portugal
}

\begin{abstract}
The electromagnetic mode spectrum of single-layer graphene subjected to a quantizing magnetic field is computed taking into account intraband and interband contributions to the magneto-optical conductivity. We find that a sequence of weakly decaying quasi-transverse-electric modes, separated by magnetoplasmon polariton modes, emerge due to the quantizing magnetic field. The characteristics of these modes are tuneable, by changing the magnetic field or the Fermi energy.
\end{abstract}

PACS numbers: 73.20.Mf, 78.67.Wj

\section{INTRODUCTION}

The field of plasmonics has been considered the photonics milestone of the year 1998. To this choice contributed the landmark paper of Ebbesen et al. on the "extraordinary optical transmission through sub-wavelength hole arrays." [1] The effect was explained on the basis of the properties of surface-plasmon polaritons. [2]

Surface-plasmon polaritons (SPP) are electromagnetic surface waves, guided by a metallic interface, resulting from the the coupling of the electromagnetic field to the collective plasma excitations of the metal.[2] These guided modes are of importance in fields as different as light guides at the nanoscale, 3] spectroscopy and sensing, enhancement of light absorption in solar cells, enhanced Raman spectroscopy, and others.

Graphene, being an one-atom-thick metallic film, is an obvious candidate for investigations on SPP. Recent research has established plasmon-based enhanced Raman spectroscopy and photocurrent, [4, 5] as well as roomtemperature prominent absorption peaks in the terahertz spectral range, [6] and nanoscopy of mid-infrared radiation confinement. [7]

Both in the two-dimensional (2D) electron gas and in graphene, the plasmon dispersion has a square-root dependence on the wave vector: $\Omega_{2 \mathrm{D}}(\boldsymbol{q}) \propto \sqrt{q}$. The linear dispersion of the electrons in graphene, $\epsilon(\boldsymbol{q})= \pm v_{F} q$, where $v_{F}$ is the Fermi velocity, implies that $\Omega_{2 \mathrm{D}}(\boldsymbol{q}) \propto$ $\sqrt{k_{F} q}$, where $k_{F} \propto \sqrt{n_{e}}$ is the Fermi momentum and $n_{e}$ is the electronic density. 8] If a grid of period $L$ is superimposed on graphene, plasmons of wave number $q \sim 1 / L$ can be excited. 2, 9] Furthermore, since $\Omega_{2 \mathrm{D}}(\boldsymbol{q}) \propto \sqrt{k_{F} q}$, we expect the scaling relation $\Omega_{2 \mathrm{D}}(\boldsymbol{q}) \propto n_{e}^{1 / 4} L^{-1 / 2}$, which has been observed experimentally. [6]

Graphene has a number of advantages over other metallic thin films used in plasmonics; e.g., the ability of changing its carrier concentration using a gate, allowing a fine control over the frequency range for plasmonic excitations, [7] and long propagation lengths as compared to conventional SPP. 10, 11] Furthermore, inhomogeneous doping in a single graphene sheet allows the drawing of SPP propagation paths. 3]

When an external magnetic field perpendicular to a $2 \mathrm{D}$ electron gas is applied, hybridization between cyclotron excitations and plasmons occurs, originating magnetoplasmon modes. 12 15] The presence of the magnetic field gives rise to strong absorption peaks, making the dispersion of electromagnetic modes very sensitive to the frequency. In this work, we demonstrate that in addition to magnetoplasmon polaritons (MPP), graphene in a magnetic field supports extremely weakly damped modes, which due to their resemblance to conventional transverse electric modes, are here referred to as quasitransverse-electric (QTE). This paper is organized as follows: In Sec. [II we overview the dispersion relation of electromagnetic modes supported by 2D electron systems. We revisit the simpler problem of zero external field, for which two types of modes can exist: SPP and weakly damped modes (transverse electric) with characteristics similar to photons propagating in a dielectric. The magneto-optical response of graphene in the presence of a quantizing magnetic field is described in Sec.III. The full mode dispersion (MPP and QTE modes) is calculated in the presence of disorder by employing the optical limit approximation to the conductivity. The losses, confinement, and polarization of the solutions are studied carefully. The outlook and conclusions are presented in Sec. IV Finally, technical details and derivations are given in appendices.

\section{DISPERSION RELATION}

We consider an infinite graphene film in the $x y$ plane embedded in a dielectric medium of permittivity (permeability) $\epsilon(\mu)$. 16] A static quantizing magnetic field is applied along the transverse $(z)$ direction. We focus on electromagnetic modes propagating along the $x$ axis,

$$
\boldsymbol{E}(\boldsymbol{r}, t)=\boldsymbol{E}_{0} e^{i(q x-\Omega t)} e^{-\kappa|z|} .
$$

The symbols have the usual meanings: $\Omega$ denotes the angular frequency, $q$ is the complex longitudinal wave 
vector, and $\kappa$ encodes the amount of confinement along the transverse direction. Maxwell equations relate these quantities according to the general relation $q^{2}=\kappa^{2}+$ $\epsilon \mu \Omega^{2}$, so that in general both $q$ and $\kappa$ are complex quantities. Note that a similar equation holds for the magnetic field $\boldsymbol{H}(\boldsymbol{r}, t)$. Throughout, we employ SI units and the notation $z=z^{\prime}+i z^{\prime \prime}$ for complex variables.

The dispersion relation of electromagnetic modes follows from the boundary conditions for the fields at the interface $z=0$ (Ref. 12):

$$
\operatorname{det}\left(\begin{array}{cc}
\frac{i \kappa \sigma_{L}(q, \Omega, B)}{2 \epsilon \Omega}+1 & \frac{\mathcal{Z}}{2} \sigma_{H}(q, \Omega, B) \\
\frac{\mathcal{Z}}{2} \sigma_{H}(q, \Omega, B) & \frac{i \mu \Omega \sigma_{L}(q, \Omega, B)}{2 \kappa}-1
\end{array}\right)=0
$$

where $B$ is the intensity of the magnetic field, $\mathcal{Z}=\sqrt{\mu / \epsilon}$ is the impedance of the surrounding medium and $\sigma_{L}\left(\sigma_{H}\right)$ denotes the longitudinal (Hall) conductivity of graphene. The physical solutions of the above equation, $q=q(\Omega)$, contain the full mode spectrum of the system - a derivation of the dispersion relation is given in Appendix A. The characteristics of the mode spectrum are determined by the conductivity tensor. The latter depends on $q, \Omega$, $B$, and, generally, also on the Fermi energy $E_{F}$, temperature and sample-specific broadening parameters. In the present work, we neglect the dependence of $\sigma_{L(H)}$ on the in-plane wave vector $q$, and thus, hereafter, $\sigma_{H(L)}$ denotes the optical (local) limit of the dynamical conductivity, i.e., $\sigma_{H(L)} \equiv \sigma_{H(L)}(\Omega, B)=\sigma_{H(L)}(0, \Omega, B)$. The latter is justified for small wave vectors, more precisely for $q l_{B} \ll 1$, where $l_{B}=\sqrt{\hbar / e B}$ denotes the magnetic length $(-e<0$ is the electron charge).

In the absence of an external field $B$, the solutions of Eq. (2) are the so-called transverse electric and transverse magnetic modes, namely, $\kappa=i \Omega \mu \sigma_{L}(\Omega, 0) / 2$ and $\kappa=2 i \Omega \epsilon / \sigma_{L}(\Omega, 0)$, respectively. In the former, the electric field is perpendicular to the direction of propagation, the mode dispersion is close to the light line, $q^{\prime}(\Omega) \simeq \Omega / c$, and damping is small $\left(q^{\prime \prime}, \kappa^{\prime} \ll q^{\prime}\right)$. Transverse electric modes require a negative reactive conductivity, $\sigma_{L}^{\prime \prime}(\Omega, 0)<0$, and hence are not observed in conventional $2 \mathrm{D}$ gases (we note that when $\sigma_{L}^{\prime \prime}<0$ and $\sigma_{L}^{\prime} /\left|\sigma_{L}^{\prime \prime}\right| \ll 1$, the behavior of the system resembles that of a dielectric). On the other hand, transverse magnetic waves are confined to the metallic surface, featuring large-field localization, thus having important applications in sub-wavelength optics and plasmonics. 17] In graphene, both modes can exist because near the interband threshold, $\Omega=2 E_{F} / \hbar$, the function $\sigma_{L}^{\prime \prime}(\Omega, 0)$ changes sign. [18] Coupling and guiding of transverse electric modes by graphene in zero field have been recently reported in Ref. 19 .

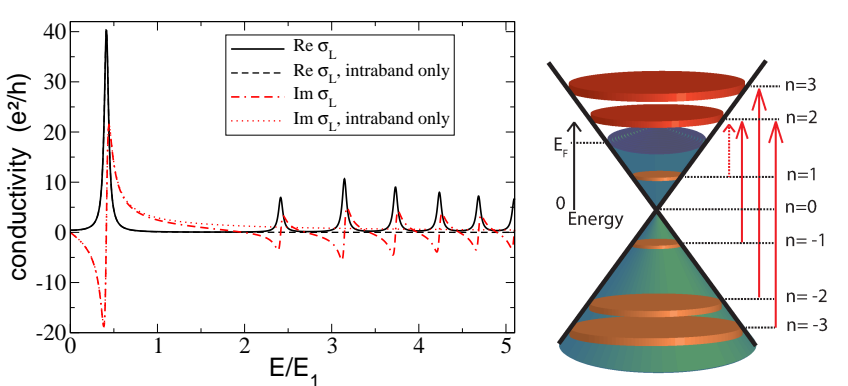

Figure 1: Doped graphene in a magnetic field at zero temperature $\left(N_{F}=1\right)$. Left: $\sigma_{L}$ is plotted as a function of $E / E_{1}$, with $E=\hbar \Omega$ and $E_{1}=E_{1}(B)$. Clearly, $\sigma_{L}^{\prime \prime}<0$ in various energy intervals. The full quantum calculation is seen to be crucial for energies above $\sim 2 E_{1}$. We have taken $\Gamma=0.03 E_{1}(B)$, which, for $B$ in the range 1-10 T, is consistent with experimental values of $\Gamma$ (see text). Right: The first few electronic transitions are shown. The Fermi energy lies slightly above the Landau level with $n=1$, and thus only transitions to levels with $n \geq 2$ are allowed. The (small) dashed arrow stands for the intraband transition responsible for the strong peak observed near at $(\sqrt{2}-1) E_{1} \sim 0.4 E_{1}$. Other arrows represent interband transitions.

\section{MAGNETO-OPTICAL MODES}

When a magnetic field is turned on, electrons acquire considerable cyclotronic energies via the Lorentz force, and at sufficiently high fields, the continuum Dirac quasiparticle spectrum condensates into degenerated Landau levels (LLs) [see Fig. 1 (right panel)], with energies given by $E_{n}(B)=s_{n} \sqrt{2|n|} \hbar v_{F} / l_{B}$; here, $n$ is the LL index $(n=0, \pm 1, \pm 2, \ldots), s_{n} \equiv \operatorname{sign}(n)$, and $v_{F} \simeq 10^{6} \mathrm{~m} / \mathrm{s}$ denotes the Fermi velocity of carriers in graphene.

We would like to investigate how the zero-field mode spectrum changes due to the quantizing magnetic field. To this end, we employ linear-response theory within the Dirac cone approximation to obtain an expression for $\sigma_{L(H)}(\Omega, B)$ with both intraband and interband contributions included (see Appendix B). In order to account for disorder, we have used an energy-independent LL broadening $\Gamma$ with ratios $\Gamma / E_{1}$ consistent with the values 1 $10 \mathrm{meV}$ found in pump-probe experiments performed in epitaxial and exfoliated graphene samples, [20, 21] and on infrared spectroscopy studies of the Drude conductivity of graphene. 22] The renormalization of the optical conductivity due to the electron-phonon interactions is neglected. From the theoretical studies, taking into account the $E_{2 g}$ optical mode at $\left.200 \mathrm{meV}, 23,24\right]$ we expect the latter approximation to be valid for frequencies below that of the optical phonon branch.

The features of $\sigma_{L}(\Omega, B)$ for doped graphene in a quantizing field are determined by the amount of disorder and the LL occupancy number of the graphene sample. The latter is defined as $N_{F}=\operatorname{int}\left[\left(E_{F} / E_{1}(B)\right)^{2}\right] \geq 0$ and yields the number of occupied (empty) electron- (hole- 


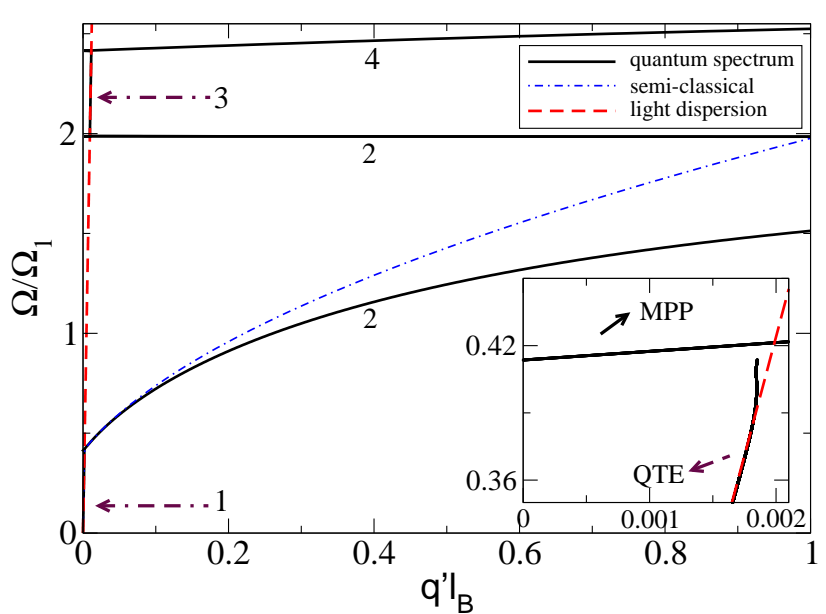

Figure 2: Mode frequency $\Omega$ for graphene in vacuum is plotted as a function of the wave vector $q^{\prime}$ for doped graphene [solid (black) line]. The wave vector $q^{\prime}$ is given in units of the inverse of the magnetic length. For completeness, semi-classical solution [dashed-dotted (blue) line] and the light dispersion [dashed (red) line] are shown. Inset: Mode spectrum near at QTE-MPP transition at $\Omega \simeq 0.4 \Omega_{1}$. The numbers in the main panel identify the distinct branches up to $\Omega \simeq 2.5 \Omega_{1}$. For consistency with the local approximation to the conductivity, we have restricted our plots to the region with $q^{\prime}(\Omega) l_{B} \leq 1$. A considerable modification to the spectrum is expected for $q^{\prime} \sim l_{B}^{-1}$ due to non-local effects in the dynamical conductivity (see text). Other parameters as in Fig. 1

) degenerate LLs for $E_{F}>0\left(E_{F}<0\right)$. In Fig. 1] (left panel), we plot $\sigma_{L}(\Omega, B)$ as a function of energy $\hbar \Omega$. These curves have $N_{F}=1$, thus covering a widerange of $E_{F}$ and $B$ values. (For concreteness, throughout, our plots refer to systems with $N_{F}=1$, except for one occasion. We complement the discussion with analytic expressions that can be used to compute the several quantities for arbitrary $N_{F}$.) The magneto-optical conductivity is seen to consist of an intraband term with spectral weight located at the lower end of the spectrum [the strong peak located at $\hbar \omega \simeq\left(\sqrt{N_{F}+1}-\sqrt{N_{F}}\right) E_{1}$ ] and interband high-frequency contributions originating a series of peaks above the interband threshold, $E \simeq$ $2 \sqrt{N_{F}} E_{1}$. The number of peaks depends on $N_{F}$ (e.g., for $N_{F}=2$, the first interband peak seen in Fig. 1 is suppressed due to Pauli blocking) and their shape depends also on $\Gamma$. For a comprehensive discussion of the magneto-optical response of graphene, see Refs. 25, 26.

In $2 \mathrm{D}$ electron gases, plasmons and cyclotronic excitations (with frequency $\omega_{\mathrm{c}}$ ) hybridize leading to the wellknown semi-classical magnetoplasmon spectrum, $\Omega^{2}=$ $\Omega_{2 \mathrm{D}}^{2}+\omega_{\mathrm{c}}^{2} \cdot[12$, 13, 27] In view of the strong contribution of interband transitions to $\sigma_{L}^{\prime \prime}$ (e.g., see the discrepancy between the semi-classical calculation and the quantum formula, even at low frequencies, in the left panel of Fig. 1), this formula should be of limited applicability in graphene. Moreover, Shubnikov-de Haas oscilla-
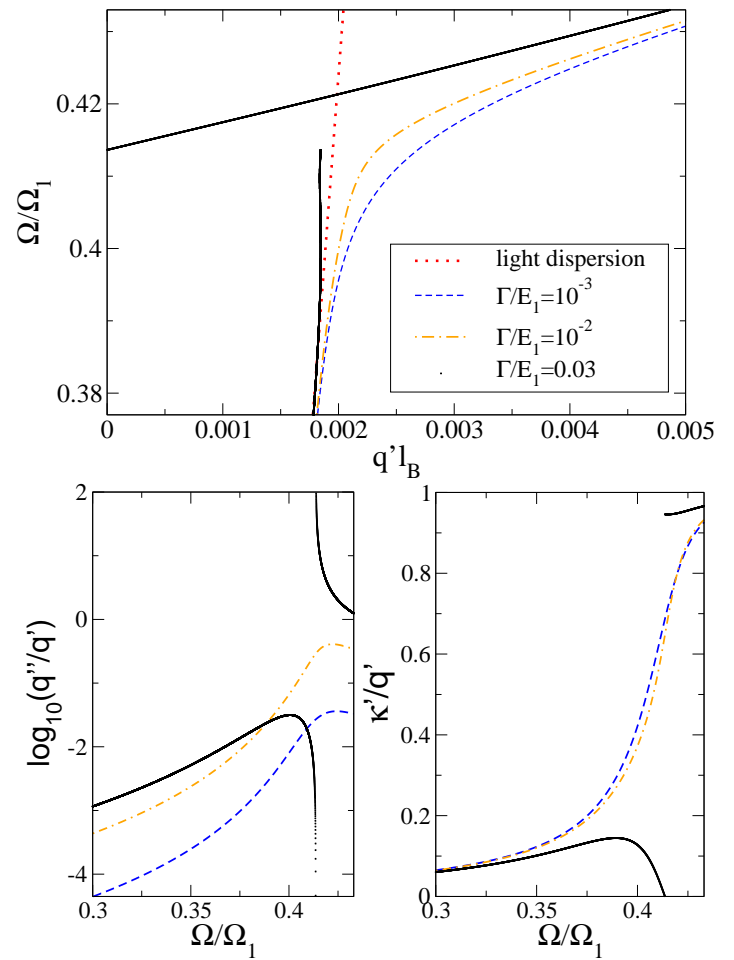

Figure 3: Mode spectrum and decay properties near a QTEMPP transition. Top panel: The dispersion relation is plotted near at $\Omega \simeq 0.4 \Omega_{1}$ for several broadening values (indicated in the legend). Bottom panel: The ratios $q^{\prime \prime}(\Omega) / q^{\prime}(\Omega)$ and $\kappa^{\prime}(\Omega) / q^{\prime}(\Omega)$ are shown for the same parameter region in the top panel. For ease of visualization, the range for the vertical axis in left panel is limited. Other parameters as in Fig. 2

tions originate many frequency regions with $\sigma_{L}^{\prime \prime}<0$, and hence, similarly to the 2D magnetized electron Fermi gas, [12, 28] we may expect the splitting of the mode spectrum into many branches.

The optical-limit solution of the dispersion relation for graphene in vacuum in the presence of the magnetic field is given in Appendix C [Eq. (28)]. The respective mode spectrum is shown in Fig. 2, with frequency given units of the LL energy scale $\Omega_{1} \equiv E_{1} / \hbar$. Note that $q^{\prime}=q^{\prime}(\Omega)$ has been plotted in the horizontal axis which helps visualizing the dispersion relation. Only non-exponentially growing solutions $\kappa^{\prime}(\Omega)>0$ are shown. The most notorious feature is the existence of a series of well-defined branches, labeled by the integers $n=1,2, \ldots$, etc. These branches can be divided into two distinct sets, namely, the set of branches with dispersion close to the light line ( $n$ odd) and the remaining ( $n$ even). The former will be shown to have the basic properties of transverse electric modes and hence are termed QTE, whereas the latter are MPP modes (with polarization not necessarily similar to transverse magnetic modes).

Our results borne out two peculiar features of graphene: i) $n$ even branches have two distinct solutions for each wave vector $q^{\prime}$. This degeneracy is a result of 
hybridization between even and odd modes, and ii) the frequency domain size of each branch is non-uniform due to the structure of LLs in graphene. The first branch occupies a region $\left[0, \tilde{\Omega}_{1}\left[\right.\right.$, the second $\left[\tilde{\Omega}_{1}, \tilde{\Omega}_{2}[\right.$, etc., where $\tilde{\Omega}_{n}$ is defined to be the $n$-th node of the reactive longitudinal conductivity, $\sigma_{L}^{\prime \prime}\left(\tilde{\Omega}_{n}, B\right)=0$. For the system under discussion, the first two nodes read $\tilde{\Omega}_{1} \simeq 0.4 \Omega_{1}$ and $\tilde{\Omega}_{2} \simeq 2 \Omega_{1}$ (see Fig. (1).

Let us now discuss with detail the intraband region $\left(\Omega \lesssim \Omega_{1}\right)$ spanning two branches, $n=1$ and $n=2$. Here, the magneto-optical transport is predominantly semiclassical, and hence the relevant frequency scale is the cyclotron frequency, $\omega_{\mathrm{c}}=e v_{F}^{2} B /\left|E_{F}\right|$. For $\tilde{\Omega}_{1}>\Omega>0$, the dispersion curve is pinned to the light dispersion line, $q^{\prime}(\Omega) \simeq \Omega / c$, except for frequencies approaching $\tilde{\Omega}_{1}$ (Fig. (2). The detachment of the QTE mode from the light line signals the onset of a rapid increase of $\sigma_{L}^{\prime \prime}$, as a result of an absorption peak in the vicinity of $\omega_{\mathrm{c}}$. A simple formula for the transition frequency $\tilde{\Omega}_{1}$ can be obtained by approximating $\sigma_{L}$ by its semi-classical value [29]

$$
\tilde{\Omega}_{1} \approx \sqrt{\omega_{\mathrm{c}}^{2}-\Gamma^{2} / \hbar^{2}} .
$$

Using the above expression, we obtain $\tilde{\Omega}_{1} \approx 0.4 \Omega_{1}$ in good agreement with the exact numerical results.

The properties of the electromagnetic modes are specially sensitive to electronic disorder close to the frequencies at which the transitions occur, since the conductivity is strongly dependent on $\Gamma$ in the vicinity of $\Omega=\tilde{\Omega}_{n}$. A closer look to the region with $\Omega \sim \tilde{\Omega}_{1}$ is provided in Fig. [ 3 for several values of $\Gamma$. This figure shows that for small broadening the transition at $\tilde{\Omega}_{1}$ can be continuous. This is further elucidated in the lower panel of Fig. [3. containing a study of $\kappa^{\prime}(\Omega)$ and $q^{\prime \prime}(\Omega)$; these quantities measure the $2 \mathrm{D}$ confinement and longitudinal losses, respectively. For the smallest values of $\Gamma$ considered, we clearly observe a smooth transition from a weakly decaying mode (typical of transverse electric modes) to a confined mode with considerable losses (typical of SPP and MPP). A remark is in order: near at $\tilde{\Omega}_{1}$, the $n=1$ (QTE) mode for $\Gamma=0.03 E_{1}$ clearly displays superluminal group velocities. The latter is a manifestation of anomalous dispersion, for which the concept of group velocity no longer describes signal propagation. [30] We believe that the velocity of signal propagation in the anomalous region equals its upper bound value, $c$, given that the mode is essentially undamped $\left(q^{\prime \prime}\right.$ and $\kappa^{\prime}$ reaching $\left.10^{-5} q^{\prime}\right)$.

The simultaneous presence of the two distinct branches $(n=1,2)$ in the intraband region $\left(\Omega \lesssim \Omega_{1}\right)$ for a given broadening value can only occur for sufficiently high fields, $B>B_{c} \equiv\left|E_{F}\right| \Gamma / e \hbar v_{F}^{2}$, otherwise one obtains a single type of solution with plasmon character, $\Omega \sim \sqrt{q^{\prime}}$, as for $B=0$. [31, 32] The reason is that for $B<B_{c}$, the reactive part of the semi-classical longitudinal conductivity is always positive below the interband threshold, thus forbidding the existence of QTE modes.

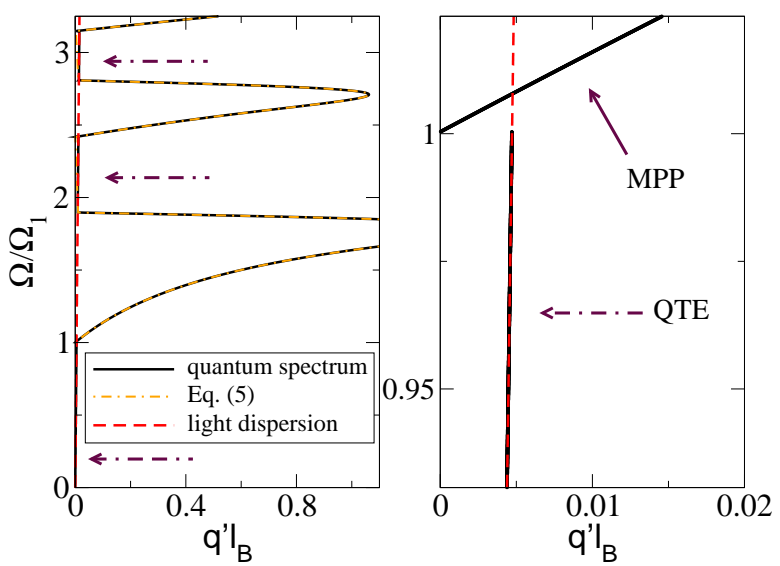

Figure 4: Mode spectrum for undoped graphene in a magnetic field $\left(N_{F}=0\right)$ [solid (black) line]. We have taken $\Gamma=0.12 E_{1}$ in this figure. The light dispersion is shown in the dashed (red) line. Arrows in the left panel indicate the first three QTE modes.

The semi-classical expression for the MPP dispersion $\Omega_{\text {(MPP) }}\left(q^{\prime}\right)$ can be derived assuming $T=0$ and ignoring the interband contributions to the magneto-optical conductivity,

$$
\Omega_{(\mathrm{MPP})}\left(q^{\prime}\right) \simeq \sqrt{\left[\Omega_{2 \mathrm{D}}\left(q^{\prime}\right)\right]^{2}+\omega_{\mathrm{c}}^{2}},
$$

where $\Omega_{2 \mathrm{D}}\left(q^{\prime}\right)=(e / \hbar) \sqrt{q^{\prime}\left|E_{F}\right| /(2 \pi \epsilon)}$ is the graphene's plasmon-polariton dispersion in zero field. The above expression is valid for $q^{\prime} \gg \Omega / c$, and for $\Omega$ within the regime of validity of the semi-classical transport theory, $\Omega \ll 2 E_{F} / \hbar$ (see dotted-dashed line in the main panel of Fig. 21). Equation. (4) predicts an increase of MPP's frequency due to the presence of a magnetic field. A derivation of the above formula is given in Appendix C. This result coincides with the semi-classical magnetoplasmon spectrum for a 2D electron gas [12, 13] and it has been obtained in Ref. 27 by studying the polarizability of graphene in a magnetic field.

We now turn our attention to the high-frequency part of the spectrum, where new branches $(n=3,4, \ldots)$ emerge due to interband transitions. Figure 2 shows that the quantum calculation (solid line) deviates considerably from the semi-classical result already at $\Omega \approx \Omega_{1}$. In particular, the quantum corrections cause a considerable slow down of the MPP's group velocity, $v_{\mathrm{g}}=\left[d q^{\prime} / d \Omega\right]^{-1}$, relative to its semi-classical value. This effect comes from the superposition of interband resonances tails that contribute with substantial weight even well below the interband threshold. For instance, in the range $\Omega / \Omega_{1} \approx$ $[1,1.5]$, the interband terms yield a correction to the conductivity of about $\sim 0.5 i e^{2} / h$ (see Fig. (1) explaining the bending of the solid curve relatively to the dashed-dotted curve in Fig. 2. Near at $\Omega=\tilde{\Omega}_{2} \approx 2 \Omega_{1}, \sigma_{L}^{\prime \prime}$ changes sign again, and a large-bandwidth $\left(\approx 0.5 \Omega_{1}\right)$ QTE mode develops. The first two QTE modes are indicated by arrows 
in Fig. 2. Their dispersion relation is well approximated by $q^{\prime}(\Omega) \simeq \Omega / c$, except within the QTE/MPP crossovers $\left(\Omega \approx \tilde{\Omega}_{n}\right.$, with $n$ odd), where $q^{\prime}(\Omega)$ acquires a complex form due the strong variation of the optical properties induced by sharp absorption peaks (see discussion above).

The full dispersion relation for the MPP branches is rather cumbersome because, as noted above, away from the semi-classical region, many interband terms contribute to the spectral weight around a particular frequency; see Eq. (20) and text therein. A compact expression for $q(\omega)$ valid for every MPP branch can still be obtained by considering $T=0$ and neglecting the Hall conductivity term in Eq. (2). These approximations are justified since (i) for a quantizing magnetic field, the conductivity of graphene does not vary significantly with temperature, and (ii) $\sigma_{H}$ can be shown to provide a small correction only in the vicinity of each $\tilde{\Omega}_{n}$. We obtain,

$$
\begin{aligned}
\sqrt{q^{2}-\epsilon \mu \Omega^{2}} & \simeq \frac{i \epsilon \Omega h}{e^{2}}\left(\frac{\Gamma / E_{1}(B)}{1-i \hbar \Omega / \Gamma}\right) \Psi^{-1}(\Omega, B), \\
\Psi(\Omega, B) & =\sum_{n=\left|N_{F}\right|}^{N_{\text {cut }} \prime} \frac{E_{1}(B) / \Delta_{n}(B)}{(1-i \hbar \Omega / \Gamma)^{2}+\Delta_{n}(B)^{2} / \Gamma^{2}} .
\end{aligned}
$$

In the above, $\Delta_{n}(B)$ stands for the $n$-th interband resonance energy, defined as $\Delta_{n}(B) \equiv E_{n+1}(B)+E_{n}(B)$. The prime in the summation sign indicates that if $N_{F} \neq$ 0 , the first term is to be halved. Also, a cutoff $n \leq$ $N_{\text {cut }}$ must be taken when computing this summation (see Appendix B). For simplicity, the above expression for $\Psi(\Omega, B)$ only includes the interband contribution to $\sigma_{L}$. The inclusion of the intraband spectral weight [see Eq. (24)] is straightforward and plays a role only in the first MPP branch. Figure 4 shows that the MPP spectrum computed from Eq. (5) can not be distinguished from the full calculation. The latter agreement extends down to low frequency (the first MPP branch) because the system plotted in Fig. 4 is half-filled (i.e., $N_{F}=0$ ). Indeed, according to our definition of interband and intraband contributions to the conductivity (see Appendix B), $\Psi(\Omega, B)$ already contains the full spectral weight.

We have discussed the general features of the mode spectrum of graphene under a quantizing electromagnetic field. It has been shown to consist of several branches, with two possible types of modes. In what follows, we demonstrate that MPP modes have conventional decaying properties of SPP, whereas the QTE modes are essentially non-decaying, with electric field nearly transverse (hence their name). The MPP solutions will be shown to have a rich polarization diagram without a clear transverse magnetic character.

\section{Decaying and polarization properties}

The decaying properties of the modes are summarized in Fig. 5. QTE modes display large localization length in
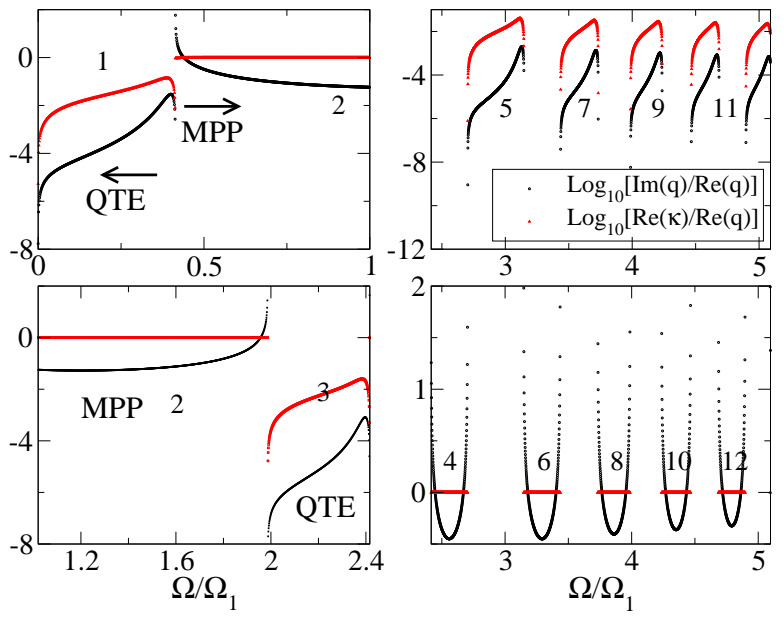

Figure 5: The ratios $q^{\prime \prime} / q^{\prime}$ and $\kappa^{\prime} / q^{\prime}$, which characterize the longitudinal and transverse decaying rates, respectively, are plotted in logarithmic scale as a function of the mode frequency. Several panels are given to help in visualizing the difference between QTE and MPP modes. (System parameters as in Fig. 1])

$x(z)$ direction, namely, $q^{\prime \prime} / q^{\prime}\left(\kappa^{\prime} / q^{\prime}\right)$ in the range $10^{-7}$ $10^{-2}\left(10^{-5}-10^{-1}\right)$ (see for instance the first branch; top left panel). MPP modes, on the other hand, always show considerable decay along the $z$ direction, $\kappa^{\prime}=\mathcal{O}\left(q^{\prime}\right)$, indicating strong confinement. The losses in the propagation direction $x$, on the other hand, vary appreciably and are determined by the graphene's absorption at the specific MPP frequency; e.g., in the range $0.4 \Omega_{1}-1.0 \Omega_{1}$, the longitudinal decay rate $q^{\prime \prime}$ varies in the range $0.1 q^{\prime}-100 q^{\prime}$, with maximum loss occurring near at the cyclotron frequency $\left(\simeq 0.4 \Omega_{1}\right)$, where the MPP lies to the left of the light line (see top panel in Fig. 3).

Although the losses and confinement reported here have orders of magnitude comparable to those in the absence of a magnetic field[10], the strong dependence of these quantities on the frequency is exclusive to the $2 \mathrm{D}$ interface subjected to a strong external magnetic field (Fig. [5 shows that the decay characteristics can vary by several orders of magnitude around at $\Omega=\tilde{\Omega}_{n}$ for all $n$.) An important effect of the magnetic field is to allow for QTE modes with lower losses than the zero-field transverse electric mode in specific frequency intervals; for instance, above $2.5 \Omega_{1}, q^{\prime \prime} / q^{\prime}$ can reach a minimum value of the order of $10^{-7}$, whereas for $B=0$ (and $E_{F} \sim 0.1 \mathrm{eV}$ ) its ratio is about $\sim 10^{-5}$.

In order to complete our study, we demonstrate that the electric field of QTE modes are essentially transverse and study how the longitudinal (transverse magnetic) character of MPP modes depend on the wave vector. To this end, we compute the ratios $\mathcal{E}_{x y(z y)} \equiv E_{x(z)} / E_{y}$ and $\mathcal{B}_{x y(z y)} \equiv B_{x(z)} / B_{y}$. Combining Maxwell equations and 
the dispersion relation, Eq. (2), we easily obtain,

$$
\begin{aligned}
\mathcal{E}_{x y} & =\left(\sigma_{L}-\frac{1}{i \omega} \frac{2 \kappa}{\mu}\right) / \sigma_{H}, \\
\mathcal{B}_{x y} & =i \frac{\kappa}{\omega}\left(2-\frac{\kappa}{i \omega \epsilon} \sigma_{L}\right) / \mu \sigma_{H},
\end{aligned}
$$

and $\mathcal{E}(\mathcal{B})_{z y}=[\operatorname{sign}(z) i q / \kappa] \mathcal{E}(\mathcal{B})_{x y}$, where it is assumed $B>0$ so that $\sigma_{H} \neq 0$. These quantities are plotted in Fig. 6] for a frequency range spanning the $n=1$ and $n=2$ branches. Below $0.4 \Omega_{1}$, the electric components ratios $\mathcal{E}_{x y(z y)}$, plotted in the right panel, are found to have magnitude in the range $10^{-3}-10^{-2}\left(10^{-2}-10^{-1}\right)$, confirming that the electric field of QTE modes lies prominently along the $y$ axis, resembling pure transverse electric modes (which have $\mathcal{E}_{x y(z y)}=0$ ). Similar conclusions can be drawn for the remaining QTE branches.

The polarization of MPP modes is found to lack a clear longitudinal character. Let us focus the branch $n=2$. For $\Omega \lesssim \Omega_{1}$ (see left panel of Fig. 6), well below the MPPQTE transition at $\Omega=\tilde{\Omega}_{2} \simeq 2 \Omega_{1}$, the magnetic ratios $\mathcal{B}_{x y(z y)}$ have values in the range 0.5 -1, making the polarization of these modes distinct from transverse magnetic (which have $\mathcal{B}_{x y(z y)}=0$ ). For $\Omega \gtrsim \Omega_{1}$, the magnetic ratio $\mathcal{B}_{x y}$ decreases with increasing frequency $/ q^{\prime}$, until it reaches a minimum at $\Omega / \Omega_{1} \simeq 1.73$ of about 0.05 (not shown). The other MPP branches display similar behavior: a strong variation of polarization near at the transitions, but with $\mathcal{B}_{x y(z y)}$ never reaching negligible values.

We have found no evidence for MPP modes with $\mathcal{B}_{x y(z y)} \approx 0$ for other choices of LL occupancy and broadening, as well. In 2D electron gases, the situation is very distinct, since large wave-vector modes are essentially longitudinal. [12, 28] A question that deserves further investigation is whether the effect of finite $\boldsymbol{q}=q^{\prime} \boldsymbol{e}_{x}$ in $\sigma_{L}$ (and $\sigma_{H}$ ) can influence the solutions at large $q^{\prime}$. A considerable renormalization of spectrum is expected for the largest wave vectors presented in our plots, which are of the order of $l_{B}^{-1}$ (see, e.g., Fig. 2). The lack of longitudinal character of MPP reported here may indeed result from an inadequacy of the optical limit in describing large $q^{\prime}$ modes. On the other hand, the system represented in Fig. 4 displays modes with smaller wave vectors [note that branches with $n>2$ have $q^{\prime}(\Omega) \lesssim l_{B}^{-1}$ ], making the optical limit less restrictive in this case. Nevertheless, similar features are observed in this system, thus providing further evidence for the generality of the phenomenons discussed in this paper, at least for small values of $q^{\prime} l_{B}$.

\section{MPP wave localization}

We briefly address the wave-localization characteristics of the MPP waves reported here. It is a well-established fact that SPP in a metal can have wavelengths considerably smaller than electromagnetic waves of the same
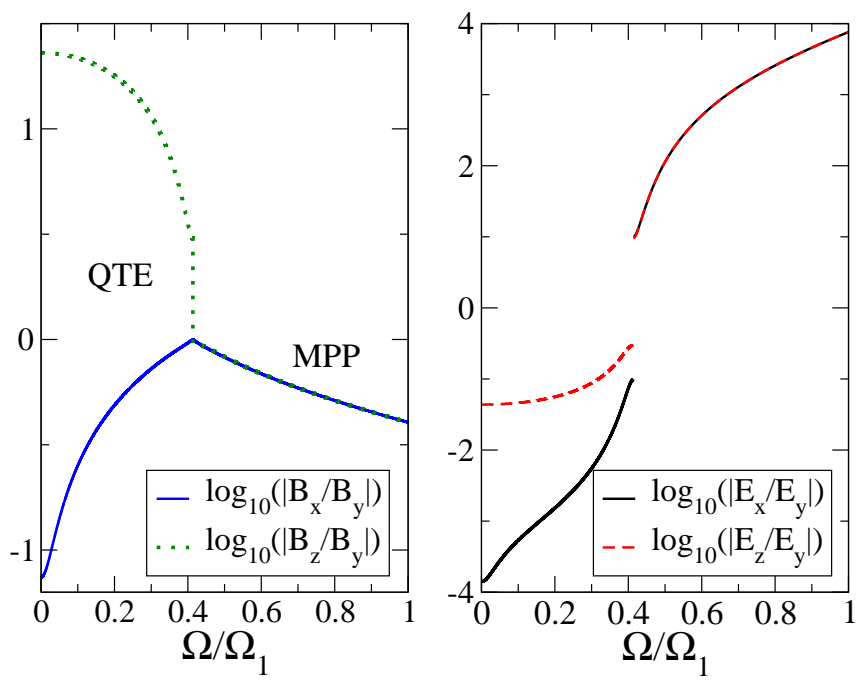

Figure 6: Polarization properties of the electromagnetic modes within the first two branches. The left panel shows the complex modulus of the magnetic ratios $\mathcal{B}_{x y}$ and $\mathcal{B}_{z y}$, whereas the respective electric counterparts, $\mathcal{E}_{x y}$ and $\mathcal{E}_{z y}$, are given in the right panel. (System parameters as in Fig. 1)

frequency in a dielectric. [17] In graphene in zero field, this shrinkage effect is enhanced when compared to conventional 2D electron gas SPP. 10, 11] Figure [7 shows the ratio of the wavelength in vacuum to the MPP mode wavelength, $\Lambda=\lambda_{0} / \lambda$ (here, $\lambda_{0}=2 \pi c / \Omega$ and $\lambda=2 \pi / q^{\prime}$ ). Near the frequency resonant to the first interband transition at $\Omega \simeq 2 \Omega_{1}$ (see also Fig. 1), we obtain a large peak of about $\Lambda \sim 10^{3}$, a figure comparable to that obtained in zero field. 10 The remaining MPP modes show peaks with $\Lambda \sim 10^{2}$. QTE solutions, on the other hand, have $\Lambda \simeq 1$ regardless of their frequency, a characteristic of transverse electric modes.

A simple formula for $\Lambda$, valid in the intraband frequency region, can be derived from Eq. (4):

$$
\Lambda \approx \frac{1}{2 \alpha E_{F}}\left(\hbar \Omega-\frac{[\Delta(B)]^{2}}{\hbar \Omega}\right),
$$

where $\alpha=e^{2} /(4 \pi \epsilon \hbar c)$ denotes the effective fine-structure constant and $\Delta(B)=\hbar e v_{F}^{2} B / E_{F}$ is the cyclotron energy. The above expression predicts a decrease of $\Lambda$ with the magnetic field, which is consistent with the exact numerical results in Fig. 7 (recall that $\Omega_{1}=\sqrt{2} v_{F} / l_{B}$ ). In the limit of $B \rightarrow 0$, Eq. (9) reproduces the result reported in Ref. [11].

\section{OUTLOOK AND CONCLUSIONS}

We have computed the spectrum of electromagnetic modes supported by a graphene interface in the presence of a quantizing magnetic field. We have found a rich structure with extended crossovers between quasi- 


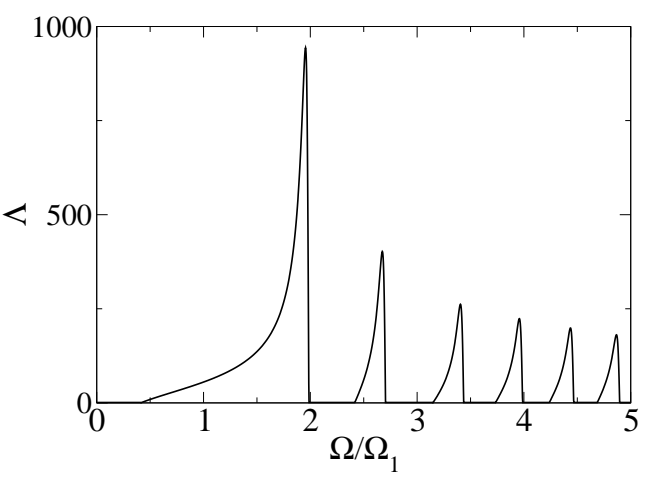

Figure 7: The wave localization ratio $\Lambda$ is plotted as a function of the mode's frequency. Other parameters as in Fig. 1

transverse-electric (QTE) and magnetoplasmon polariton (MPP) modes as a consequence of characteristic Shubnikov-de Haas oscillations in the magneto-optical response of graphene. Analogously to the 2D Fermi gas, the dispersion relation splits in a large number of branches. [12, 28] Interband transitions between the bottom and top Dirac cones originate terms in the conductivity with considerable spectral weight in the semi-classical (low-frequency) region. As a consequence, the conventional semi-classical 2D magnetoplasmon dispersion becomes restricted to a narrow wave-vector/frequency interval. Our calculation within the optical-limit approximation to the conductivity predicts that, unlike 2D Fermi gases, MPP modes with a given wave vector admit two possible values of frequency in the same MPP branch. The consequences of a non-zero wave vector $\boldsymbol{q}$ in the conductivity for the QTE-MPP spectrum is a challenging question and deserves further investigation.

In summary, we have shown that a quantizing magnetic field changes the conventional picture of electromagnetic modes in graphene. 10, 18] In the LL regime, the mode spectrum splits into many branches, consisting of magnetoplasmon polaritons (MPP) and quasitransverse-electric (QTE) modes. At small frequencies, a QTE persists, even in the semi-classical regime, as long as the cyclotronic frequency is larger than the electrons relaxation's rate. Due to the high LL energy gaps, these effects should be observable up to room temperature.

\section{ACKNOWLEDGEMENTS}

This work was supported by the NRF-CRP award "Novel 2D materials with tailored properties: beyond graphene" (R-144-000-295-281).

\section{APPENDIX A: MODE SPECTRUM}

For our purposes it is sufficient to consider a single Fourier component of the electromagnetic field in the following form

$$
\boldsymbol{E}_{m}=\left(E_{m, x}, E_{m, y}, E_{m, z}\right) e^{i\left(q_{m} x-\omega t\right)} e^{-\kappa_{m}|z|},
$$

where $\kappa_{m}$ accounts for possible attenuation in the transverse direction to graphene. The subscript $m=1(2)$ denotes the region of space with $z>0(z<0)$. In this section, we consider the more general case of graphene embedded in dielectric mediums with permittivities $\epsilon_{1}$ and $\epsilon_{2}$.

We require $\kappa_{m}^{\prime} \geq 0$ and $q_{m}^{\prime \prime} / q_{m}^{\prime} \geq 0$. The former means that the wave can be confined to the $z$ plane and must not diverge as $z \rightarrow \pm \infty$, whereas the second condition allows wave attenuation in the $x$ direction. The formal divergence at $x \rightarrow-\infty$ is a consequence of the beginning of the perturbation at $t=-\infty$; for a detailed discussion on the built-in divergence of leaky waves in the context of SPP see Ref. 33 .

The macroscopic Maxwell equations imply the following relation between the field amplitudes,

$$
i \omega \overrightarrow{\mathcal{F}_{m}}=M_{m} \overrightarrow{\mathcal{F}_{m}}
$$

where $\overrightarrow{\mathcal{F}_{m}}$ is the six-dimensional vector of amplitudes $\overrightarrow{\mathcal{F}_{m}}=\left(\boldsymbol{E}_{m}, \boldsymbol{B}_{m}\right)$ and,

$$
M_{m}=\left[\begin{array}{cc}
0 & M_{m, R} \\
M_{m, L} & 0
\end{array}\right],
$$

with

$$
\begin{aligned}
& M_{m, R}=\frac{1}{\epsilon_{m} \mu_{m}}\left[\begin{array}{ccc}
0 & (-1)^{m} \kappa_{m} & 0 \\
(-1)^{m+1} \kappa_{m} & 0 & i q_{m} \\
0 & -i q_{m} & 0
\end{array}\right] \text {, } \\
& M_{m, L}=\left[\begin{array}{ccc}
0 & (-1)^{m+1} \kappa_{m} & 0 \\
(-1)^{m} \kappa_{m} & 0 & -i q_{m} \\
0 & i q_{m} & 0
\end{array}\right] \text {. }
\end{aligned}
$$

A straightforward consequence of Eq. (11) is the wellknown relation,

$$
q_{m}^{2}=\kappa_{m}^{2}+\epsilon_{m} \mu_{m} \omega^{2} .
$$

The mode spectrum for this problem is derived by imposing the boundary conditions for the electromagnetic field at the interface $z=0$ and making use of the relations between the field components [Eqs. (11)-(14)]. The continuity of the tangential (normal) component of the electric field (magnetic induction) implies that $q_{1}=q_{2} \equiv q$, $E_{1, x(y)}=E_{2, x(y)}$ and $B_{1, z}=B_{2, z}$. The discontinuity of the tangential component of the magnetic field yields

$$
\begin{aligned}
& \frac{1}{\mu_{1}} B_{1, y}-\frac{1}{\mu_{2}} B_{2, y}=-\sigma_{x x} E_{1, x}-\sigma_{x y} E_{1, y}, \\
& \frac{1}{\mu_{1}} B_{1, x}-\frac{1}{\mu_{2}} B_{2, x}=\sigma_{y x} E_{1, x}+\sigma_{y y} E_{1, y},
\end{aligned}
$$


where $\sigma_{\alpha \beta} \equiv \sigma_{\alpha \beta}(\boldsymbol{q}, \omega)[\alpha, \beta=x, y]$ denotes the dynamical conductivity of graphene,

$$
\sigma_{\alpha \beta}(\boldsymbol{q}, \omega)=\frac{J_{s, \alpha}(\boldsymbol{q}, \omega)}{E_{s, \beta}(\boldsymbol{q}, \omega)},
$$

and relates the Fourier transforms of the surface current, $J_{s, \alpha}(\boldsymbol{q}, \omega)$, and that of the electric field, $E_{s, \beta}(\boldsymbol{q}, \omega)$, at $z=$ 0 . The use of the local limit of the conductivity $\sigma_{\alpha \beta}(\boldsymbol{q}=$ $0, \omega)$ is justified whenever the wave vectors of interest are much smaller than the inverse of typical length scales. In the presence of a quantizing magnetic field, the local limit is justified for $|\boldsymbol{q}| \ll l_{B}^{-1}$, where $l_{B}$ denotes the magnetic length (see Appendix B).

Combining the above results, it is straightforward to obtain the general dispersion relation

$$
\begin{aligned}
\frac{i \omega}{\kappa_{1}} \epsilon_{1}[ & \left.\left(1+\frac{\kappa_{1}}{\kappa_{2}} \frac{\epsilon_{2}}{\epsilon_{1}}\right)-\sigma_{L} \frac{\kappa_{1}}{i \omega \epsilon_{1}}\right] \times \\
\times & {\left[\sigma_{L}-\left(\frac{\kappa_{1}}{\mu_{1}}+\frac{\kappa_{2}}{\mu_{2}}\right) \frac{1}{i \omega}\right]=\sigma_{H}^{2} . }
\end{aligned}
$$

In order to obtain the above form, we have invoked rotational symmetry and used the notation employed in the main text: $\sigma_{L} \equiv \sigma_{x x}$ and $\sigma_{H} \equiv \sigma_{x y}$. Setting $\epsilon_{1}\left(\mu_{1}\right)=\epsilon_{2}\left(\mu_{2}\right) \equiv \epsilon(\mu)$ (and hence $\kappa_{1}=\kappa_{2} \equiv \kappa$ ) leads to the Eq. (2) in the main text. We remark that the term with $\sigma_{H}$ is negligible for most choices of parameters. We have verified that only near at the QTE/MPP transitions, where $\sigma_{L}^{\prime \prime} \simeq 0$, the Hall conductivity $\sigma_{H}$ provides a (small) correction to the spectrum.

\section{APPENDIX B: MAGNETO-OPTICAL CONDUCTIVITY OF GRAPHENE}

Within the Dirac-cone approximation, [8] and modeling the effect of disorder by an energy broadening function, the magneto-optical conductivity of graphene at Fermi energy $E_{F}$ and temperature $T$ assumes the simple form in the random phase approximation [25, 26]

$$
\begin{aligned}
\sigma_{L(H)}(\omega, B) & =g_{s} g_{v} \times \frac{e^{2}}{4 h} \times \\
& \times \sum_{n \neq m} \frac{\Xi_{L(H)}^{n m}}{i \Delta_{n m}} \frac{n_{F}\left(E_{n}\right)-n_{F}\left(E_{m}\right)}{\hbar \omega+\Delta_{n m}+i \Gamma_{n m}(\omega)},
\end{aligned}
$$

where $g_{s(v)}=2$ is the spin (valley) degeneracy factor of graphene, $n_{F}(E)=1 /\left[1+e^{\left(E-E_{F}\right) / k_{B} T}\right]$ stands for the Fermi distribution function, $\Gamma_{n m}(\omega)$ is the LL broadening, $\Delta_{n m}=E_{n}-E_{m}$, with LL energies $E_{n}$ given by

$$
E_{n}=\operatorname{sign}(n)\left[\hbar v_{F} / l_{B}\right] \sqrt{2|n|},
$$

with $l_{B}$ denoting the magnetic length, $l_{B} \equiv \sqrt{\hbar /(e B)}$, $v_{F} \simeq 10^{6} \mathrm{~m} / \mathrm{s}$ is the Fermi velocity, and

$$
\begin{aligned}
& \Xi_{L}^{n m}=\frac{\hbar^{2} v_{F}^{2}}{l_{B}^{2}}\left(1+\delta_{m, 0}+\delta_{n, 0}\right) \delta_{|m|-|n|, \pm 1}, \\
& \Xi_{H}^{n m}=i \Xi_{L}^{n m}\left(\delta_{|m|,|n|-1}-\delta_{|m|-1,|n|}\right) .
\end{aligned}
$$

The use of the low-energy (Dirac-cone approximation) theory to compute $\sigma_{L(H)}$ assumes an infinite sea of negative energy states, and thus requires a cutoff $|n|,|m| \leq$ $N_{\text {cut }}$ in Eq. (20). The respective cutoff energy $E_{N_{\text {cut }}}$ is of the order of graphene's bandwidth. Results are largely insensitive to the precise value chosen for the cutoff; in our numerical calculations we have considered $E_{N_{\text {cut }}}=3 \mathrm{eV}$.

The magneto-optical conductivity of graphene has two types of terms: i) intraband contributions corresponding to transitions within the same Dirac cone (i.e., $n=m \pm$ 1), and ii) interband transitions that couple the valence and conduction Dirac cones (i.e., $n=-m \pm 1$ ). Transitions involving the zero energy LL (e.g., $n=0 \rightarrow n=1$ ) need to be considered separately because this LL state contains electrons and holes. Here, for convenience, we classify the transitions involving the zero energy LL as interband-like.

The general expression Eq. (20) can be put into a more useful form by separating interband and intraband contributions (with the proviso made in the previous paragraph). For the sake of simplicity, we assume $T=0$ and $E_{F} \geq 0$ [the conductivity for holes can be obtained using the symmetry relations: $\sigma_{L}\left(-E_{F}\right)=\sigma_{L}\left(E_{F}\right)$ and $\left.\sigma_{H}\left(-E_{F}\right)=-\sigma_{H}\left(E_{F}\right)\right]$. We denote the number of occupied electron LLs by $N_{F}$, that is, $N_{F}=\operatorname{int}\left[\left(E_{F} / E_{1}\right)^{2}\right] \geq$ 0 . Intraband transitions $\left(n=N_{F} \rightarrow n=N_{F}+1\right)$ involve an energy difference of $\Delta_{\text {intra }}=\sqrt{2} \hbar v_{F} / l_{B}\left(\sqrt{N_{F}+1}-\right.$ $\left.\sqrt{N_{F}}\right)$. Its contribution to the conductivity dominates at small frequencies where most of the spectral weight is concentrated around $\omega=\Delta_{\text {intra }} / \hbar$. The intraband (semiclassical) conductivity therefore consists of a single term in the summation Eq. (20), reading

$$
\begin{aligned}
\sigma_{L}^{\text {(intra) }} & =\frac{2 e^{2}}{h} \frac{\hbar^{2} v_{F}^{2}}{l_{B}^{2} \Gamma \Delta_{\text {intra }}} \frac{1-i \hbar \omega / \Gamma}{(1-i \hbar \omega / \Gamma)^{2}+\Delta_{\text {intra }}^{2} / \Gamma^{2}}, \\
\sigma_{H}^{\text {(intra) }} & =-\frac{2 e^{2}}{h} \frac{\hbar^{2} v_{F}^{2}}{l_{B}^{2} \Gamma^{2}} \frac{1}{(1-i \hbar \omega / \Gamma)^{2}+\Delta_{\text {intra }}^{2} / \Gamma^{2}} .
\end{aligned}
$$

According to our classification the latter equations are valid for $N_{F} \neq 0$, otherwise there is no intraband contribution. Note that for high Fermi energy/low magnetic field, one recovers the familiar semi-classical Drude conductivity [Eqs. (33) and (34)], since the cyclotronic gap $\Delta_{\text {intra }}$ equals the cyclotronic energy $\hbar \omega_{\mathrm{c}}$ when many levels are occupied, $N_{F} \gg 1$. 26.

Interband terms dominate at frequencies close or above the interband threshold, $\omega=2 E_{F} / \hbar$. These transitions involve the energy difference energy $\Delta_{n} \equiv$ $\sqrt{2} \hbar v_{F} / l_{B}(\sqrt{n+1}+\sqrt{n})$, with $n \geq N_{F}$. Its contribu- 
tion to the magneto-optical conductivity reads as

$$
\begin{aligned}
\sigma_{L}^{(\text {inter })=} & \frac{2 e^{2}}{h} \frac{\hbar^{2} v_{F}^{2}}{l_{B}^{2}} \sum_{n=N_{F}}^{N_{\text {cut }}}\left(1+\delta_{n, 0}\right)\left(2-\delta_{n, N_{F}}\right) \times \\
& \times \frac{1}{\Gamma \Delta_{n}} \frac{1-i \hbar \omega / \Gamma}{(1-i \hbar \omega / \Gamma)^{2}+\Delta_{n}^{2} / \Gamma^{2}}, \\
\sigma_{H}^{(\text {inter })}= & -\frac{2 e^{2}}{h} \frac{\hbar^{2} v_{F}^{2}}{l_{B}^{2} \Gamma^{2}} \frac{1+\delta_{N_{F}, 0}}{(1-i \hbar \omega / \Gamma)^{2}+\Delta_{N_{F}}^{2} / \Gamma^{2}} .
\end{aligned}
$$

\section{APPENDIX C: SEMI-CLASSICAL DISPERSION}

We now derive an approximate formula for the semiclassical MPP dispersion. The first step is to solve Eq. (2) for $\kappa(\Omega)$; we obtain

$$
\kappa(\Omega)=\frac{X(\Omega) \pm \sqrt{4 f(\Omega) g(\Omega)+X(\Omega)^{2}}}{2 f(\Omega)},
$$

with the notation,

$$
\begin{aligned}
f(\Omega) & =\frac{i}{2 \Omega \epsilon} \sigma_{L}(\Omega, B), \\
g(\Omega) & =\frac{i \Omega \mu}{2} \sigma_{L}(\Omega, B), \\
X(\Omega) & =-1+f(\Omega) g(\Omega)-\frac{\mu}{4 \epsilon} \sigma_{H}^{2}(\Omega, B) .
\end{aligned}
$$

The sign in the numerator in Eq. (28) must be chosen according to the requirements necessary to obtain a physical solution (Appendix A). The complex wave vector $q(\omega)$ follows from Eq. (15),

$$
q(\Omega)=\sqrt{\kappa(\Omega)^{2}+\epsilon_{\mathrm{r}} \Omega^{2} / c^{2}},
$$

where $\epsilon_{\mathrm{r}}$ denotes the relative permittivity of the dielectric medium surrounding graphene. [16] In order to proceed, we neglect the effect of interband transitions and approximate Eqs. (24) and (25) by their semi-classical analog [26]

$$
\begin{aligned}
\sigma_{L} & =\frac{e^{2}}{h} \frac{2\left|E_{F}\right|}{\Gamma} \frac{1-i \hbar \Omega / \Gamma}{(1-i \hbar \Omega / \Gamma)^{2}+\Delta^{2} / \Gamma^{2}}, \\
\sigma_{H} & =-\frac{e^{2}}{h} \frac{2 E_{F}}{\Gamma} \frac{\Delta / \Gamma}{(1-i \hbar \Omega / \Gamma)^{2}+\Delta^{2} / \Gamma^{2}},
\end{aligned}
$$

where $\Delta=\hbar e v_{F}^{2} B /\left|E_{F}\right|$ is the intraband cyclotron gap. The semi-classical expressions have the advantage of simplifying the notation and introducing the cyclotron energy which is more used in the literature (albeit less accurate than the intraband gap, $\Delta_{\text {intra }}$; see Appendix B). The crucial point to derive a compact expression for the dispersion relation is to note that for typical frequencies $\sim \mathrm{THz}$ and $\Gamma \sim 0.01 \mathrm{eV}$, we have $f \times g \ll X^{2}$, and therefore the expression for $\kappa(\Omega)$ can be approximated by

$$
\kappa(\Omega) \simeq \frac{X(\Omega)}{f(\Omega)}
$$

where we have chosen the appropriate sign in Eq. (28). [Note that the other solution has $\kappa(\Omega) \simeq 0$ and would correspond to a QTE mode.] Raising both sides of Eq. (35) to the power of two, substituting the conductivity tensor components [Eqs. (33) and (34)] into $X(\Omega)$, and employing a series expansion for small $\Delta / \hbar \Omega$ and $\Gamma / \hbar \Omega$, we obtain,

$$
q^{\prime 2}+q^{\prime \prime 2} \simeq \epsilon_{\mathrm{r}} \frac{\Omega^{2}}{c^{2}}+\left(\frac{2 \pi \epsilon \hbar \Omega}{E_{F} e^{2}}\right)^{2}\left(\hbar^{2} \Omega^{2}-2 \Delta^{2}\right),
$$

where we have kept the terms up to second order in the small parameters, and assumed $\hbar \Omega / E_{F} \gg g_{0} \sqrt{\mu / \epsilon} \simeq 0.1$ (with $g_{0} \equiv 2 e^{2} / h$ denoting the quantum of conductance). We remark that these approximations are consistent with the small-wavelength limit, for which $\hbar \Omega$ is typically larger than other energy scales. Assuming negligible damping $q^{\prime \prime} \ll q^{\prime}$, more precisely, requiring

$$
\Gamma \ll \frac{E_{F}^{2} e^{4} q^{\prime 2}}{\epsilon^{2} \hbar^{3} \Omega^{3}},
$$

and taking $q^{\prime} \gg \Omega / c$ (non-retarded regime) in Eq. (36), we arrive at the final result,

$$
q^{\prime} \approx \frac{2 \pi \epsilon \hbar \Omega}{\left|E_{F}\right| e^{2}} \sqrt{\hbar^{2} \Omega^{2}-2 \Delta^{2}}
$$

The magneto-plasmon spectrum [Eq. (4)] follows immediately by expanding the latter expression in the small parameter $\Delta / \hbar \Omega$.

[1] T. W. Ebbesen, H. J. Lezec, H. F. Ghaemi, T. Thio, and P. A. Wolf, Nature 391, 667 (1998).

[2] S. A. Maier, Plasmonics: Fundamentals and Applications, Springer (2007).

[3] A. Vakil, and N. Engheta, Science 332, 1291 (2011).

[4] F. Schedin, E. Lidorikis, A. Lombardo, V. G. Kravets, A. K. Geim, A. N. Grigorenko, K. S. Novoselov, and A. C. Ferrari, ACSNano 4, 5617 (2010).

[5] T. J. Echtermeyer, L. Britnell, P. K. Jasnos, A. Lombardo, R. V. Gorbachev, A. N. Grigorenko, A. K. Geim, A. C. Ferrari, and K. S. Novoselov, Nature Communications 2, 458 (2011).

[6] L. Ju, B. Geng, J. Horng, C. Girit, M. C. Martin, Z. Hao, H. A. Bechtel, X. Liang, A. Zettl, Y. R. Shen, and F. Wang, Nature Nanotechnology 6, 630 (2011).

[7] Z. Fei, G. O. Andreev, W. Bao, L. M. Zhang, A. S. McLeod, C. Wang, M. K. Stewart, Z. Zhao, G. Dominguez, M. Thiemens, M. M. Fogler, M. J. Taube, A. H. Castro-Neto, C. N. Lau, F. Keilmann, and D. N. Basov, Nano Lett. 11, 4701 (2011).

[8] A. H. Castro Neto, F. Guinea, N. M. R. Peres, K. S. Novoselov, and A. K. Geim, Rev. Mod. Phys 81, 109 (2009).

[9] Yu. V. Bludov, M. I. Vasilevskiy, and N. M. R. Peres, EPL 92, 68001 (2010).

[10] M. Jablan, H. Buljan, and M. Soljačić, Phys. Rev. B 80, 245435 (2009). 
[11] F. H. L. Koppens, D. E. Chang, and F. J. G. de Abajo, Nano Lett. 11, 3370 (2011).

[12] K. W. Chiu, and J. J. Quinn, Phys. Rev. B 9, 4724 (1974).

[13] I. V. Kukushkin, V. M. Muravev, J. H. Smet, M. Hauser, W. Dietsche, and K. von Klitzing, Phys. Rev. B 73, 113310 (2006).

[14] Y. A. Bychkov, and G. Martinez, Phys. Rev. B 77, 125417 (2008).

[15] O. L. Berman, G. Gumbs, and Y. E. Lozovik, Phys. Rev. B 78, 085401 (2008).

[16] Usually experiments are done with graphene on top of a substrate such as $\mathrm{SiO}_{2}$ (see e.g., Ref. 7]). The presence of two distinct dielectrics can be accounted for letting $\epsilon \rightarrow\left(\epsilon_{1}+\epsilon_{2}\right) / 2$, where $\epsilon_{1(2)}$ concerns with the top (bottom) dielectric. This prescription is accurate in the non-retarted regime $q^{\prime} \gg \Omega / c$; for the exact dispersion relation we refer to Appendix A.

[17] W. L. Barnes, A. Dereux, and T. W. Ebbesen, Nature (London) 424, 824 (2003).

[18] S. A. Mikhailov, and K. Ziegler, Phys. Rev. Lett. 99, 016803 (2007)

[19] Q. Bao, H. Zhang, B. Wang, Z. Ni, C. H. Y. X. Lim, Y. Wang, D. Y. Tang, and K. P. Loh, Nature Photonics 5, 411(2011).

[20] J. M. Dawlaty, S. Shivaraman, M. Chandrashekhar, F. Rana, and M. G. Spencer, Appl. Phys. Lett. 92, 042116 (2008).

[21] M. Breusing, S. Kuehn, T. Winzer, E. Malić, F. Milde, N. Severin, J. P. Rabe, C. Ropers, A. Knorr, and T. Elsaesser, Phys. Rev. B 83, 153410 (2011).

[22] J. Horng, C.-F. Chen, B. Geng, C. Girit, Y. Zhang, Z.
Hao, H. A. Bechtel, M. Martin, A. Zettl, M. F. Crommie, Y. R. Shen, and F. Wang, Phys. Rev. B 83, 165113 (2011).

[23] A. Pound, J. P. Carbotte, and E. J. Nicol, EPL 94, 57006 (2011).

[24] A. Pound, J. P. Carbotte, and E. J. Nicol, Phys. Rev. B 85, $125422(2012)$.

[25] V.P. Gusynin, S.G. Sharapov, and J.P. Carbotte, J. Phys.: Condens. Matter 19, 026222 (2007).

[26] A. Ferreira, J. Viana-Gomes, Yu. V. Bludov, V. Pereira, N. M. R. Peres, A. H. Castro Neto, Phys. Rev. B 84, 235410 (2011)

[27] R. Roldán, J.-N. Fuchs, and M. O. Goerbig, Phys. Rev. B 80, 085408 (2009).

[28] D. C. Bardos, and N. E. Frankel, Phys. Rev. B 49, 4096 (1994).

[29] In deriving this expression we have approximated the conductivity in the intraband region by its semi-classical value. The latter is consistent since i) the intraband gap $\hbar \Omega_{1}\left(\sqrt{N_{F}+1}-\sqrt{N_{F}}\right)$ is well-approximated by the cyclotron energy $\hbar \omega_{c}$ in samples with $N_{F} \geq 1$ (see Appendix $\mathrm{B}$ ), and ii) the spectral weight in this region coming from interband transitions is negligible for $\Gamma \ll E_{1}$. We have also neglected the contribution from the Hall condutivity to the dispersion relation (see Appendix A for more details).

[30] J. D. Jackson, Classical Electrodynamics (Wiley, New York, 1999).

[31] F. Stern, Phys. Rev. Lett. 18, 546 (1967).

[32] K. W. K. Shung, Phys. Rev. B 34, 979 (1986).

[33] N. E. Glass, M. Weber, and D. L. Mills, Phys. Rev. B 29, 6548 (1984). 